\title{
Predicting Strength Ratio of Laminated Composite Material with Evolutionary Artificial Neural Network
}

\author{
Huiyao Zhang ${ }^{1}$, Atsushi Yokoyama ${ }^{2}$ \\ Department of Fiber Science and Engineering \\ Kyoto Institute of Technology \\ Kyoto, JAPAN
}

\begin{abstract}
In this paper, an alternative methodology to obtain the strength ratio for the laminated composite material is presented. Traditionally, classical lamination theory and related failure criteria are used to calculate the numerical value of strength ratio of laminated composite material under in-plane and out-ofplane loading from a knowledge of the material properties and its layup. In this study, to calculate the strength ratio, an alternative approach is proposed by using an artificial neural network, in which the genetic algorithm is proposed to optimize the search process at four different levels: the architecture, parameters, connections of the neural network, and active functions. The results of the present method are compared to those obtained via classical lamination theory and failure criteria. The results show that an artificial neural network is a feasible method to calculate the strength ratio concerning in-plane loading instead of classical lamination and associated failure theory.
\end{abstract}

Keywords-Classical lamination theory; genetic algorithm; artificial neural network; optimization

\section{INTRODUCTION}

Fiber-reinforced composite materials have gained increasing attention due to their superior mechanical performance in stiffness, strength, and specific gravity of fibers over conventional materials. Laminated composite material takes advantage of fiber-reinforced composite material, and finds wide application in a variety of applications, which include electronic packaging, sports equipment, homebuilding, medical prosthetic devices, high-performance military structures, etc. The mechanical properties of composite laminated are determined by stacking sequence, ply thickness, fiber orientation, and material for each ply. Strength ratio[1], [2], [3], [4], [5], [6], [7], [8] is a critical index to predict the performance of a laminated composite material. There are two approaches for solving this problem: analytical methods, such as classical lamination theory(CLT); data-driven methods, such as artificial neural networks (ANN).

The analytical approach involves a two-step procedure to obtain strength ratio: first, develop the stress and strain relationship among in-plane loading using classical lamination theory based on a knowledge of the composite laminate properties of the individual layers and the laminate geometry; then calculate the strength ratio according to associated failure criteria, such as Tsai-Wu failure criterion, based on the aboveobtained stress and strain relationship. However, the use of CLT needs intensive computation since it involves massive matrix multiplication and integration operation.

The other approach to this problem is using an artificial neural network, which is a data-driven method, instead of an analytical method. ANN, heavily inspired by biology and psychology, is a reliable tool instead of a complicated mathematical model, which can accelerate the calculation process and reduce the computation cost. It has been widely used to solve various practical engineering problems in applications [9], [10], such as pattern recognition, nonlinear regression, data mining, clustering, prediction, etc. Evolutionary artificial neural networks are a subclass of artificial neural networks, in which evolutionary algorithms are introduced to design the topology of an ANN. For an artificial neural network, the number of layers, the connection between neurons, the activation functions used in every neuron, etc. are critical components to its performance. The design of an ANN can be treated as an optimization procedure of discrete variables, which can be solved by a genetic algorithm (GA). It is claimed that the combinations of artificial neural networks and evolutionary algorithm [11] can significantly improve the performance of intelligent systems than that rely on ANNs or evolutionary algorithms alone.

GA, inspired by Darwin's principle of survival of the fittest, is widely adopted to obtain the global optimal for discrete optimization problems. The techniques used in this algorithm, such as selection, crossover, mutation, are derived from natural selection, and individuals with better fitness get more chances to breed. Therefore, GA can be integrated into the design of ANN, in which encoding the information of an artificial neural network into a chromosome [12], [13].

The rest of this paper is organized as the following: Section II introduces the CLT and the failure criteria, which is used to check whether the composite material fails or not in the present study; Section III covers the design of an artificial neural network for a function approximation; Section IV reviews the use of the genetic algorithm in the design of neural network architecture, and the techniques of parameters optimization during the training process; Section V presents the result of the numerical experiments in different cases; in the conclusion part, we present and discuss the experiment results.

\section{Classical LAMinAtion THEORY AND FAILURE CRITERIA}

\section{A. Classical Lamination Theory}

Classical lamination theory derives from three simplifying assumptions in laminated composite material: the laminate consist of plies bonded together through the thickness, the thickness of each ply is small, and it is consists of homogeneous, orthotropic material; the entire laminated composite 
is only under in-plane loading; the Normal cross-section of the laminate is vertical to the deflected middle surface. Fig. 1 shows the coordinate system used for an angle lamina. The axis in the 1-2 coordinate system is called the local axis or the material axis, and the axis in the $\mathrm{x}$-y coordinate system is called the global axis.

A few cases of laminates, such as symmetric laminates, cross-ply laminates, play an important role in the application of laminated composite material. A laminate is called an angle ply laminate if it has plies of the same material and thickness and is only oriented at $+\theta$ and $-\theta$ directions. A model of an angle ply laminate is as shown in Fig. 2 .

1) Stress and Strain in a Lamina: For a single lamina under in-plane loading whose thickness is relatively small, suppose the upper and lower surfaces of the lamina are free from external loading. According to Hooke's law, the threedimensional stress-strain equations can be reduced to twodimensional stress-strain equations in the composite material. The stress-strain relation in local axis 1-2 is

$$
\left[\begin{array}{l}
\sigma_{1} \\
\sigma_{2} \\
\tau_{12}
\end{array}\right]=\left[\begin{array}{ccc}
Q_{11} & Q_{12} & 0 \\
Q_{12} & Q_{22} & 0 \\
0 & 0 & Q_{66}
\end{array}\right]\left[\begin{array}{l}
\varepsilon_{1} \\
\varepsilon_{2} \\
\gamma_{12}
\end{array}\right],
$$

where $Q_{i j}$ is the stiffness of a lamina. And they are related to engineering elastic constants as follows:

$$
\begin{aligned}
Q_{11} & =\frac{E_{1}}{1-v_{12} v_{21}}, \\
Q_{22} & =\frac{E_{2}}{1-v_{12} v_{21}}, \\
Q_{66} & =G_{12}, \\
Q_{12} & =\frac{v_{21} E_{2}}{1-v_{12} v_{21}},
\end{aligned}
$$

where $E_{1}, E_{2}, v_{12}, G_{12}$ are four independent engineering elastic constants, which are defined as follows: $E_{1}$ is the longitudinal Young's modulus, $E_{2}$ is the transverse Young's modulus, $v_{12}$ is the major Poisson's ratio, and $G_{12}$ is the in-plane shear modulus.

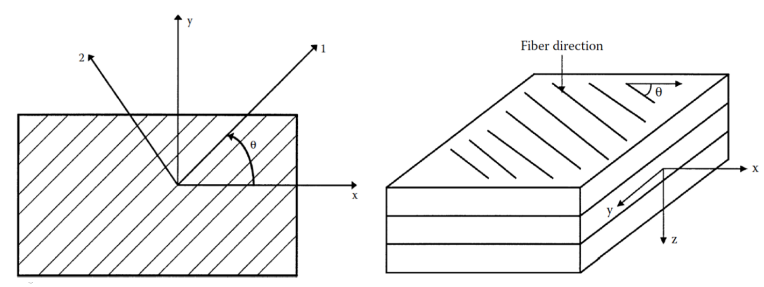

Fig. 1. The Left Diagram Shows the Local and Global Axis of an Angle Lamina, which is from a Laminate as Shown in the Right Diagram.

\begin{tabular}{c}
\hline$+\theta$ \\
\hline$-\theta$ \\
\hline$\cdots$ \\
\hline$-\theta$ \\
\hline$+\theta$ \\
\hline
\end{tabular}

Fig. 2. Model for Angle Ply Laminate.
Stress-strain relation in the global $\mathrm{x}-\mathrm{y}$ axis is

$$
\left[\begin{array}{c}
\sigma_{x} \\
\sigma_{y} \\
\tau_{x y}
\end{array}\right]=\left[\begin{array}{lll}
\bar{Q}_{11} & \bar{Q}_{12} & \bar{Q}_{16} \\
\bar{Q}_{12} & \bar{Q}_{22} & \bar{Q}_{26} \\
\bar{Q}_{16} & \bar{Q}_{26} & \bar{Q}_{66}
\end{array}\right]\left[\begin{array}{l}
\varepsilon_{x} \\
\varepsilon_{y} \\
\gamma_{x y}
\end{array}\right],
$$

where

$$
\begin{aligned}
& \bar{Q}_{11}=Q_{11} \cos ^{4} \theta+Q_{22} \sin ^{4} \theta+2\left(Q_{12}+2 Q_{66}\right) \sin ^{2} \theta \cos ^{2} \theta, \\
& \bar{Q}_{12}=\left(Q_{11}+Q_{22}-4 Q_{66}\right) \sin ^{2} \theta \cos ^{2} \theta+Q_{12}\left(\cos ^{4} \theta+\sin ^{2} \theta\right), \\
& \bar{Q}_{22}=Q_{11} \sin ^{4} \theta+Q_{22} \cos ^{4} \theta+2\left(Q_{12}+2 Q_{66}\right) \sin ^{2} \theta \cos ^{2} \theta, \\
& \bar{Q}_{16}=\left(Q_{11}-Q_{12}-2 Q_{66}\right) \cos ^{3} \theta \sin \theta-\left(Q_{22}-Q_{12}-2 Q_{66}\right) \sin ^{3} \theta \cos \theta, \\
& \bar{Q}_{26}=\left(Q_{11}-Q_{12}-2 Q_{66}\right) \cos \theta \sin { }^{3} \theta-\left(Q_{22}-Q_{12}-2 Q_{66}\right) \cos 3 \theta \sin \theta, \\
& \bar{Q}_{66}=\left(Q_{11}+Q_{22}-2 Q_{12}-2 Q_{66}\right) \sin \theta^{2} \cos ^{2}+Q_{66}\left(\sin \theta^{4}+\cos \theta^{4}\right) .
\end{aligned}
$$

2) Stress and Strain in a Laminate: For forces acting on laminates, such as in plate and shell structures, the relationship between applied forces and displacement can be given by

$$
\begin{aligned}
{\left[\begin{array}{l}
N_{x} \\
N_{y} \\
N_{x y}
\end{array}\right] } & =\left[\begin{array}{lll}
A_{11} & A_{12} & A_{16} \\
A_{12} & A_{22} & A_{26} \\
A_{16} & A_{26} & A_{66}
\end{array}\right]\left[\begin{array}{c}
\varepsilon_{x}^{0} \\
\varepsilon_{y}^{0} \\
\gamma_{x y}^{0}
\end{array}\right] \\
& +\left[\begin{array}{lll}
B_{11} & B_{12} & B_{16} \\
B_{11} & B_{12} & B_{16} \\
B_{16} & B_{26} & B_{66}
\end{array}\right]\left[\begin{array}{c}
k_{x} \\
k_{y} \\
k_{x y}
\end{array}\right],
\end{aligned}
$$

where $N_{x}, N_{y}$ refers to the normal force per unit length; $N_{x y}$ means shear force per unit length; $\varepsilon^{0}$ and $k_{x y}$ denotes mid plane strains and curvature of a laminate in $\mathrm{x}-\mathrm{y}$ coordinates The mid-plane strain and curvature is given by

$$
\begin{aligned}
A_{i j} & =\sum_{k=1}^{n}\left(\overline{Q_{i j}}\right)_{k}\left(h_{k}-h_{k-1}\right) i=1,2,6, j=1,2,6, \\
B_{i j} & =\frac{1}{2} \sum_{k=1}^{n}\left(\overline{Q_{i j}}\right)_{k}\left(h_{k}^{2}-h_{k-1}^{2}\right) i=1,2,6, j=1,2,6, \\
D_{i j} & =\frac{1}{3} \sum_{k=1}^{n}\left(\overline{Q_{i j}}\right)_{k}\left(h_{k}^{3}-h_{k-1}^{3}\right) i=1,2,6, j=1,2,6 .
\end{aligned}
$$

The $[\mathrm{A}],[\mathrm{B}]$, and $[\mathrm{D}]$ matrices are called the extensional, coupling, and bending stiffness matrices, respectively. The extensional stiffness matrix $[A]$ relates the resultant in-plane forces to the in-plain strains, and the bending stiffness matrix $[D]$ couples the resultant bending moments to the plane curvatures. The coupling stiffness matrix $[B]$ relates the force and moment terms to the midplane strains and curvatures.

\section{B. Failure Criteria for a Lamina}

Failure criteria for composite materials are more difficult to predict due to structural and material complexity. The failure process of composite materials can be regarded from microscopic and macroscopic points of view. The most popular criteria about the failure of an angle lamina are from the macroscopic point of view, which are according to the tensile, compressive, and shear strengths. As shown in Fig. 3, there are two types of failure criteria [14], [15], [16], [17], [18], [19], [20], [21] according to failure surfaces. The first failure surface is a rectangle that includes the maximum stress failure criterion [22], and maximum strain failure criterion. The second failure surface is ellipsoidal that includes Tsai-Wu [23], [24], Chamis, Hoffman, and Hill criteria. In the present study, the two most 
reliable failure criteria are adopted, Maximum stress and Tsaiwu. Both these failure criteria are based on the stress in the local axis instead of principal normal stress and maximum shear stresses, in which four normal strength parameters and one shear stress are involved. The five strength parameters are:

$\left(\sigma_{1}^{T}\right)_{u l t}=$ ultimate longitudinal tensile strength(in direction 1),

$$
\begin{aligned}
& \left(\sigma_{1}^{C}\right)_{u l t}=\text { ultimate longitudinal compressive strength, } \\
& \left(\sigma_{2}^{T}\right)_{u l t}=\text { ultimate transverse tensile strength, } \\
& \left(\sigma_{2}^{C}\right)_{u l t}=\text { ultimate transverse compressive strength, and } \\
& \left(\tau_{12}\right)_{\text {ult }}=\text { and ultimate in-plane shear strength. }
\end{aligned}
$$

1) Maximum stress (MS) failure criterion: Maximum stress failure criteria are consist of the normal stress theory and the shear stress theory. The stress applied to a lamina can be resolved into the normal stress and shear stress in the local axis. The lamina fails if either of the normal stress or shear stress in the local axis of a lamina is equal or exceeds the corresponding ultimate strengths of the unidirectional lamina. That is,

$$
\begin{array}{lll}
\sigma_{1} \geq\left(\sigma_{1}^{T}\right)_{u l t} & \text { or } & \sigma_{1} \leq-\left(\sigma_{1}^{C}\right)_{u l t} \\
\sigma_{2} \geq\left(\sigma_{2}^{T}\right)_{u l t} & \text { or } \quad \sigma_{2} \leq-\left(\sigma_{2}^{C}\right)_{u l t} \\
\tau_{12} \geq\left(\tau_{12}\right)_{u l t} & \text { or } & \tau_{12} \leq-\left(\tau_{12}\right)_{u l t}
\end{array}
$$

where $\sigma_{1}$ and $\sigma_{2}$ are the normal stresses in the local axis 1 and $2 ; \tau_{12}$ is the shear stress in the symmetry plane 1-2.

2) Tsai-Wu failure criterion: The Tsai-Wu criterion is one of the most reliable static failure criteria derived from the von Mises yield criterion. A lamina is considered to fail if

$$
\begin{aligned}
H_{1} \sigma_{1} & +H_{2} \sigma_{2}+H_{6} \tau_{12}+H_{11} \sigma_{1}^{2}+H_{22} \sigma_{2}^{2} \\
& +H_{66} \tau_{12}^{2}+2 H_{12} \sigma_{1} \sigma_{2}<1
\end{aligned}
$$

is violated, where
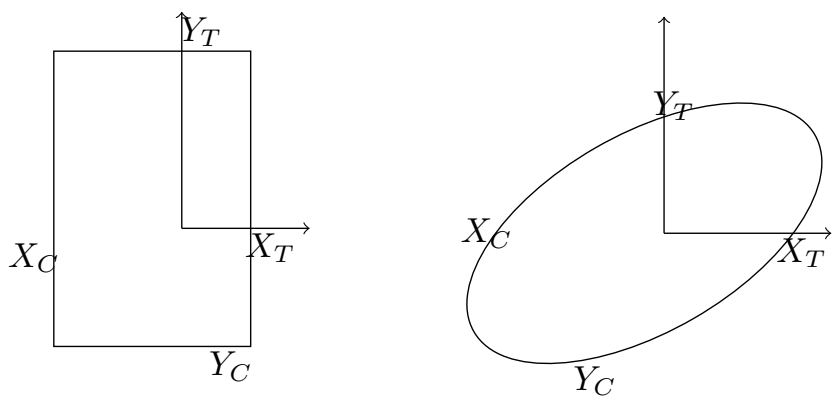

Fig. 3. Schematic Failure Surfaces for Maximum Stress and Quadratic Failure Criteria.

$$
\begin{aligned}
H_{1} & =\frac{1}{\left(\sigma_{1}^{T}\right)_{u l t}}-\frac{1}{\left(\sigma_{1}^{C}\right)_{u l t}}, \\
H_{11} & =\frac{1}{\left(\sigma_{1}^{T}\right)_{u l t}\left(\sigma_{1}^{C}\right)_{u l t}}, \\
H_{2} & =\frac{1}{\left(\sigma_{2}^{T}\right)_{u l t}}-\frac{1}{\left(\sigma_{2}^{C}\right)_{u l t}}, \\
H_{22} & =\frac{1}{\left(\sigma_{2}^{T}\right)_{u l t}\left(\sigma_{2}^{C}\right)_{u l t}}, \\
H_{66} & =\frac{1}{\left(\tau_{12}\right)_{u l t}^{2}}, \\
H_{12} & =-\frac{1}{2} \sqrt{\frac{\left(\sigma_{1}^{T}\right)_{u l t}\left(\sigma_{1}^{C}\right)_{u l t}\left(\sigma_{2}^{T}\right)_{u l t}\left(\sigma_{2}^{C}\right)_{u l t}}{u}} .
\end{aligned}
$$

$H_{i}$ is the strength tensor of the second-order; $H_{i j}$ is the strength tensor of the fourth-order. $\sigma_{1}$ is the applied normal stress in direction $1 ; \sigma_{2}$ is the applied normal stress in direction 2; $\tau_{12}$ is the applied in-plane shear stress.

3) Strength ratio: The safety factor, or yield stress, is how much extra load beyond is intended a composite laminate will take. The strength ratio(SR) is defined as

$$
S R=\frac{\text { Maximum Load Which Can Be Applied }}{\text { Load Applied }} .
$$

\section{Evolutionary Artificial Neural Network}

\section{A. General Neural Network}

In this paper, the feedforward ANN is adopted in the current study, since it is straightforward to code. For function approximation through an artificial neural network, Cybenko demonstrated that a two-layer perceptron can form an arbitrarily close approximation to any continuous nonlinear mapping [25]. Therefore, a two-layer feedforward ANN is proposed in the present study. Fig. 4 shows a general framework for a two-layer ANN, in which the number of nodes in the hidden layer and the connection with inputs, are critical in the design of an ANN. The nodes in the hidden layer are treated as feature extractors or detectors. Therefore, nodes within this layer should partially be connected with the inputs of an ANN, since the unnecessary connections would increase the model's complicacy, which will reduce an ANN's performance. The number of nodes in the hidden layer should be less than the number of inputs since the nodes in the hidden layer are features. For the nodes in the last layer, every node should be fully connected with nodes in the previous layer, the relationship between the outputs and features should be direct. The rest, which affects the performance of an artificial neural network, are the activation function, and ANNs training method. In the following section, the $i$ th node in the input layer is denoted as $i_{i}$, and the $j$ th node in the hidden layered denoted as $h_{j}$, respectively.

\section{B. Activation Function}

The activation function is one of the critical parts of an ANN. Liu [12] et al. claims that the performance of neural 


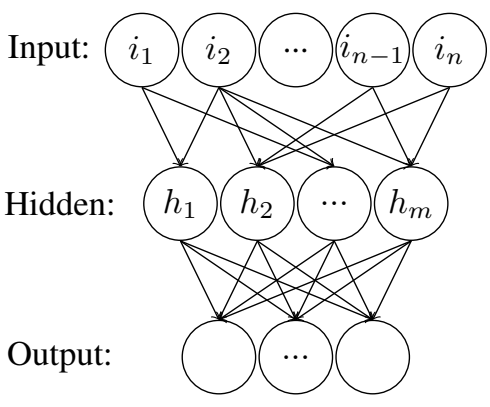

Fig. 4. Network Diagram for the Two-layer Neural Network. The Input, Hidden, and Output Variables are Represented by Nodes, and the Weight Parameters are Represented by Links between the Nodes. Arrows Denote the Direction of Information Flow Through the Network During Forward Propagation.

networks with different activation functions is different, even if they have the same architecture. A generalized activation function can be written as

$$
y_{i}=f_{i}\left(\sum_{j=1}^{n} w_{i j} x_{j}-\theta\right)
$$

where $y_{i}$ is the output of the node $i, x_{j}$ is the $j$ th input to the node, and $w_{i j}$ is the connection weight between adjacent nodes $i$ and $j$. Table I display the most widely adopted activation functions in the design of an ANN, which is used in the current study.

\section{Weights Learning}

The weight training in an ANN is to minimize the error function, such as the most widely used mean square error function, which calculates the difference between the desired and the prediction output values averaged overall examples. Gradient descent algorithm is widely adopted to reduce the value of an error function, which has been successfully applied in many practical areas. However, this class of algorithms is plagued by the possible existence of local minima or "flat spots" and "the curse of dimensionality". One method to overcome this problem is to adopt a genetic algorithm.

\section{Methodology}

For an angle ply laminate, its strength ratio can be computed based on Tsai-Wu failure theory or maximum stress theory given the laminate's lay-up, material properties, in-plane loading, etc. To model this function, we propose an ANN framework as shown in Fig. 6, which derives from the previous two-layer model. There are sixteen inputs of this ANN, which are in-plane loading $N_{x}, N_{y}$, and $N_{x y}$; design parameters of a

TABLE I. EXAMPLES OF WIDELY USED ACTIVATION FUNCTIONS IN THE Design of an ARTIFICIAL NeURAL NeTwork

\begin{tabular}{lllcc}
\hline Type & Description & Formula & Range & Encoding \\
\hline Linear & The output is proportional to the input & $f(x)=c x$ & $(-\infty,+\infty)$ & 00 \\
Sigmoid & A family of S-shaped functions & $f(x)=\frac{1}{1+e^{-c x}}$ & $(0,1)$ & 01 \\
ReLU & A piece-wise function & $f(x)=\max \{0, x\}$ & $(0,+\infty)$ & 10 \\
Softplus & A family of S-shaped functions & $f(x)=\ln \left(1+e^{x}\right)$ & $(0,+\infty)$ & 11 \\
\hline
\end{tabular}

laminate, two fiber orientation $\theta_{1}$ and $\theta_{2}$, ply thickness $t$, total number of plies $N$; five engineering constants of composite materials, $E_{1}, E_{2}, G_{12}$, and $v_{12}$; five strength parameters of a unidirectional lamina. Two outputs are strength ratio according to MS theory and strength ratio according to Tsai-Wu theory.

The work involved in the evolution process of ANN consists of three parts: search space, which includes the topology of an ANN, activation function, etc.; search strategy, which details how to explore the search space; performance estimation strategy refers to the measurement of the performance of an artificial neural network.

\section{A. Search Space}

We propose a general neural network framework as shown in Fig. 4. The search space is parameterized by four parts: (1) the number of nodes $\mathrm{m}$ (possibly unbounded) in the hidden layer, to further narrow down the search space, the assumption is $m$ less than $n$; (2) the type of operation every node executes, e.g., sigmoid, linear, Gaussian; (3) the connection relationship between the hidden nodes and inputs (4) the weight value in the connection if a connection exists.

Therefore, the evolution process in an evolutional artificial neural network can be divided into four different levels: topology, learning rules, active functions, and connection weights. For the evolution of the topology, the aim is to find an optimal ANN architecture for a specific problem. The architecture

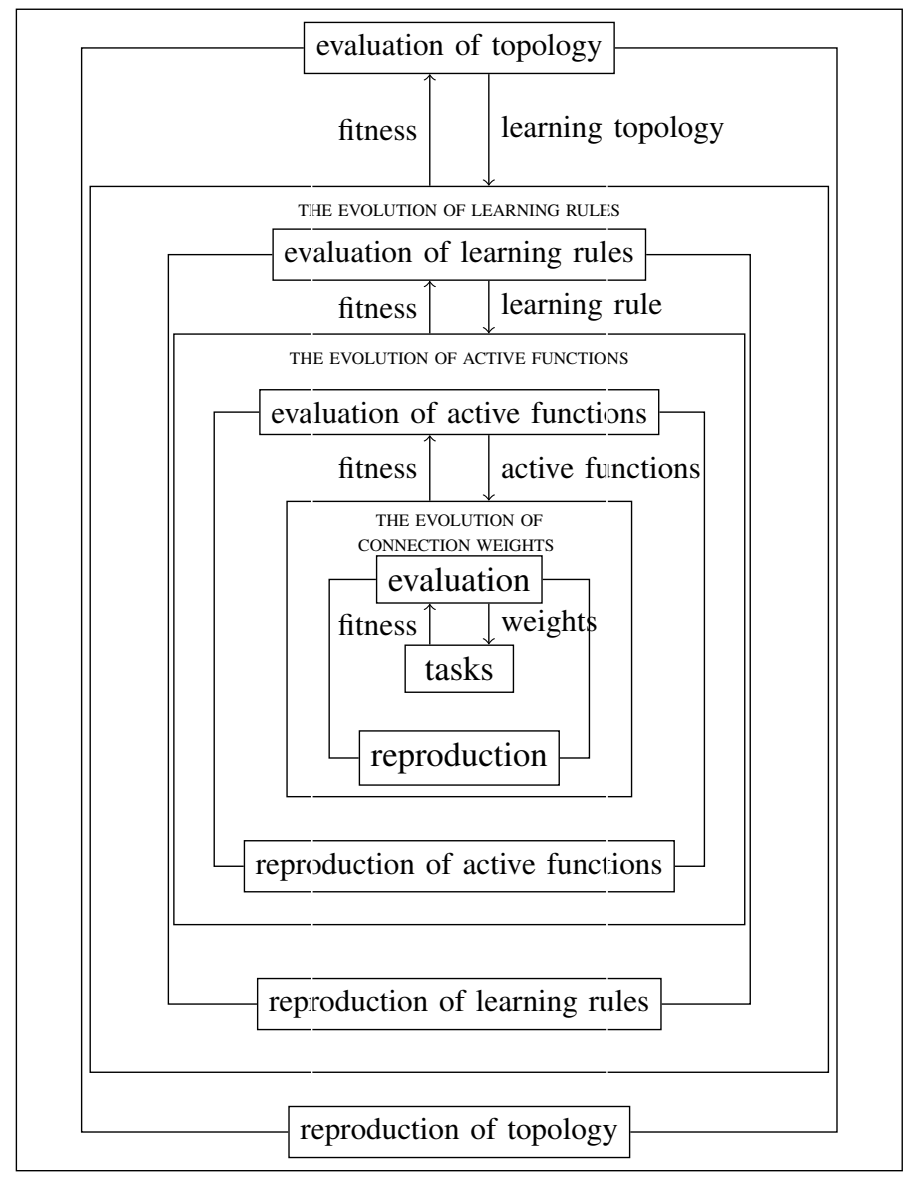

Fig. 5. A General Framework for Evolutionary Neural Network, in which Fitness Refers to the Corresponding Value of Objective Function. 


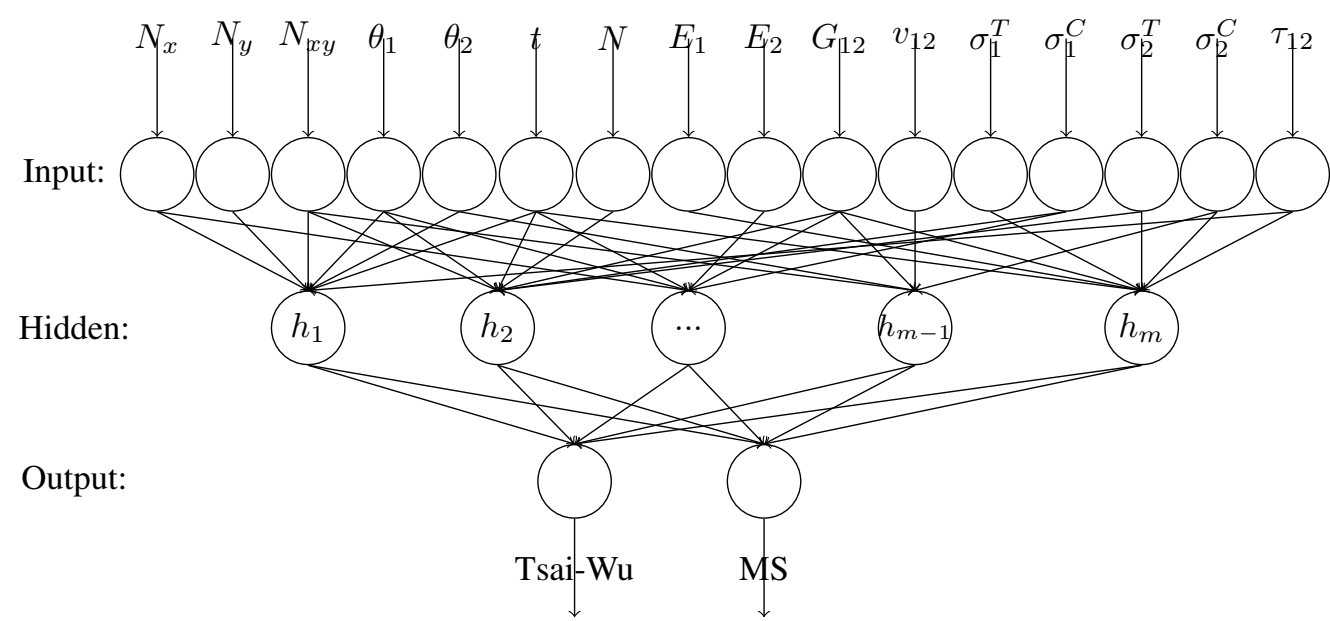

Fig. 6. Diagram for Modeling the Target Function of Strength Ratio Calculating for an Angle Ply Laminate.

TABLE II. The Binary Representation of PARent 1 , PAREnt 2 , and Child Corresponding to Fig .7(A), (B) AND (C), with $i_{1}, i_{2}, \cdots, i_{16}$

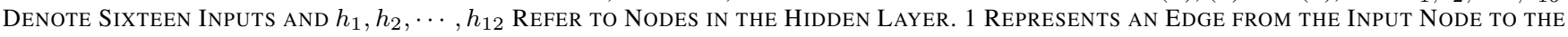
Hidden NOdE, AND 0 REPRESENTS NO EDGE FROM THE INPUT NODES TO THE HidDEN NODE.

\begin{tabular}{|c|c|c|c|c|c|c|c|c|c|c|c|c|c|c|c|c|c|c|c|}
\hline Hidden & Nodes & $i_{1}$ & $i_{2}$ & $i_{3}$ & $i_{4}$ & $i_{5}$ & $i_{6}$ & $i_{7}$ & $i_{8}$ & $i_{9}$ & $i_{10}$ & $i_{11}$ & $i_{12}$ & $i_{13}$ & $i_{14}$ & $i_{15}$ & $i_{16}$ & $\mathrm{f}$ & f \\
\hline \multirow{5}{*}{ P1 } & $h_{1}$ & 1 & 1 & 1 & 1 & 1 & 1 & 0 & 0 & 0 & 0 & 0 & 0 & 0 & 0 & 1 & 1 & 0 & 0 \\
\hline & $h_{2}$ & 0 & 1 & 1 & 1 & 0 & 0 & 0 & 1 & 0 & 0 & 1 & 1 & 0 & 0 & 0 & 0 & 1 & 1 \\
\hline & $h_{3}$ & 1 & 0 & 0 & 1 & 0 & 1 & 1 & 0 & 1 & 1 & 0 & 0 & 1 & 0 & 0 & 0 & 0 & 0 \\
\hline & $h_{4}$ & 0 & 0 & 1 & 0 & 1 & 0 & 0 & 0 & 0 & 1 & 0 & 1 & 0 & 0 & 1 & 0 & 0 & 1 \\
\hline & $h_{5}$ & 0 & 0 & 0 & 0 & 0 & 1 & 0 & 1 & 0 & 1 & 0 & 1 & 0 & 1 & 1 & 1 & 0 & 1 \\
\hline \multirow{12}{*}{ P2 } & $h_{1}$ & 0 & 0 & 0 & 0 & 0 & 0 & 1 & 1 & 1 & 1 & 1 & 0 & 0 & 0 & 0 & 0 & 1 & 0 \\
\hline & $h_{2}$ & 1 & 1 & 1 & 1 & 0 & 0 & 0 & 0 & 0 & 0 & 0 & 0 & 0 & 0 & 0 & 0 & 0 & 0 \\
\hline & $h_{3}$ & 1 & 1 & 1 & 1 & 0 & 0 & 0 & 0 & 1 & 1 & 1 & 1 & 0 & 0 & 0 & 0 & 1 & 1 \\
\hline & $h_{4}$ & 0 & 0 & 1 & 1 & 1 & 1 & 1 & 0 & 0 & 0 & 0 & 0 & 0 & 0 & 0 & 0 & 0 & 0 \\
\hline & $h_{5}$ & 0 & 0 & 0 & 0 & 0 & 0 & 0 & 0 & 0 & 0 & 0 & 1 & 0 & 1 & 1 & 1 & 0 & 1 \\
\hline & $h_{6}$ & 0 & 0 & 0 & 0 & 0 & 1 & 0 & 1 & 0 & 1 & 0 & 1 & 0 & 1 & 1 & 1 & 0 & 1 \\
\hline & $h_{7}$ & 0 & 0 & 0 & 0 & 0 & 0 & 0 & 0 & 0 & 0 & 0 & 0 & 0 & 1 & 1 & 1 & 0 & 0 \\
\hline & $h_{8}$ & 0 & 0 & 0 & 0 & 0 & 1 & 0 & 0 & 0 & 1 & 0 & 0 & 0 & 1 & 0 & 1 & 0 & 0 \\
\hline & $h_{9}$ & 0 & 0 & 0 & 0 & 0 & 1 & 0 & 1 & 0 & 1 & 0 & 1 & 0 & 0 & 0 & 0 & 0 & 1 \\
\hline & $h_{10}$ & 0 & 1 & 1 & 1 & 0 & 0 & 0 & 0 & 1 & 1 & 1 & 1 & 0 & 0 & 0 & 0 & 1 & 1 \\
\hline & $h_{11}$ & 0 & 0 & 0 & 0 & 0 & 0 & 0 & 0 & 1 & 1 & 1 & 1 & 0 & 0 & 0 & 0 & 1 & 1 \\
\hline & $h_{12}$ & 0 & 0 & 0 & 0 & 0 & 0 & 0 & 0 & 0 & 0 & 1 & 1 & 1 & 1 & 1 & 0 & 1 & 1 \\
\hline \multirow{8}{*}{ Child } & $h_{1}$ & 1 & 1 & 1 & 1 & 1 & 1 & 0 & 0 & 0 & 0 & 0 & 0 & 0 & 0 & 1 & 1 & 0 & 0 \\
\hline & $h_{2}$ & 0 & 1 & 1 & 1 & 0 & 0 & 0 & 1 & 0 & 0 & 1 & 1 & 0 & 0 & 0 & 0 & 1 & 1 \\
\hline & $h_{1}$ & 0 & 0 & 0 & 0 & 0 & 0 & 1 & 1 & 1 & 1 & 1 & 0 & 0 & 0 & 0 & 0 & 1 & 0 \\
\hline & $h_{2}$ & 1 & 1 & 1 & 1 & 0 & 0 & 0 & 0 & 0 & 0 & 0 & 0 & 0 & 0 & 0 & 0 & 0 & 0 \\
\hline & $h_{3}$ & 1 & 1 & 1 & 1 & 0 & 0 & 0 & 0 & 1 & 1 & 1 & 1 & 0 & 0 & 0 & 0 & 1 & 1 \\
\hline & $h_{4}$ & 0 & 0 & 1 & 1 & 1 & 1 & 1 & 0 & 0 & 0 & 0 & 0 & 0 & 0 & 0 & 0 & 0 & 0 \\
\hline & $h_{5}$ & 0 & 0 & 0 & 0 & 0 & 0 & 0 & 0 & 0 & 0 & 0 & 1 & 0 & 1 & 1 & 1 & 0 & 1 \\
\hline & $h_{6}$ & 0 & 0 & 0 & 0 & 0 & 1 & 0 & 1 & 0 & 1 & 0 & 1 & 0 & 1 & 1 & 1 & 0 & 1 \\
\hline
\end{tabular}

of a neural network determines the information processing capability in an application, which is the foundation of the ANN. Two critical issues are involved in the search process of an ANN architecture: the representation and the search operators. Fig. 5 summarizes these four levels of evolution in an ANN.

\section{B. Search Strategy}

It is necessary to define related operations during the GA process, which includes the representation of an artificial neural network, the fitness function that determines how good a solution is, and the search operators, such as selection, mutation, and crossover.
For the representation of an ANN, encode the $h_{i}$ node as an eighteen digits binary string. The initial sixteen digits in the string correspond to the connections between $i_{i}$ and $h_{i}$, with ' 1 ' implying there exists a connection between them, with ' 0 ' implying no connection exists. The last two digits in the string refer to an activation function, such as "01" which means a sigmoid function. Table II are examples of the binary representation of ANNs whose architectures are as shown in Fig. 7.

For the objective function, treat the multiplicative inverse of the mean squared error, which is the difference between the target and actual output averaged overall examples, as the fitness function. 


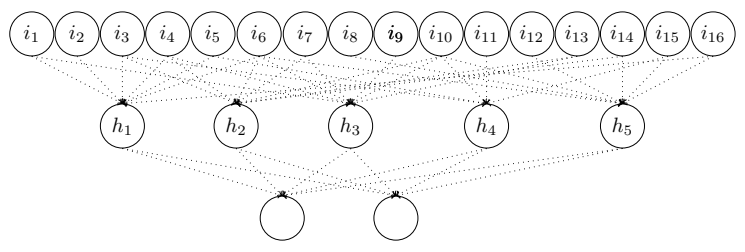

(a) Parent 1

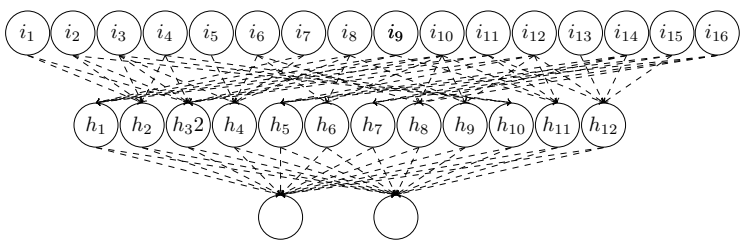

(b) Parent 2

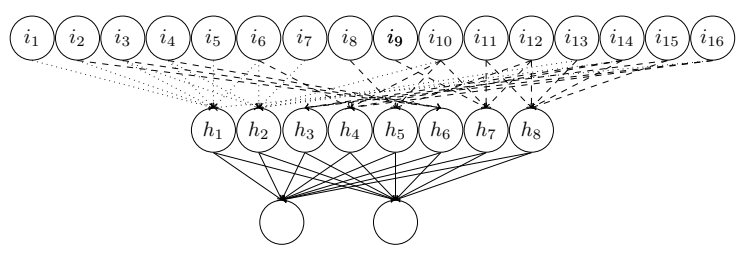

(c) Child

Fig. 7. Examples of three ANNs, with (a) and (b) as parent ANNs, and (c) as the child of (a) and (b). child $c$ inherits the connection relationship part from parent 1 denoted by the darker dashed lines,and the rest from parent 2 denoted by the gray dashed line.

The crossover between individuals results in exploiting the area between the given two-parent solutions. In the present study, we search the local area by combining the genes of half number of nodes from both parents. Fig. 7 illustrates the crossover operator: Fig. 7(c) is the child of Fig. 7(a) and Fig. 7(b), the connection relationship of hidden nodes with inputs are from both parents, and the corresponding activation functions are also from both parents. In the binary representation Table II, it is shown that the first two rows of the child are the same as the first two rows of parent $P_{1}$, and the last six rows of the child are the same as the first six rows of parent $P_{2}$.

\section{Performance Estimation Strategy}

The simplest approach to this problem is to perform standard training and validation of the architecture on a dataset. However, this method is inefficient and computationally intensive. Therefore, much recent research [26] focuses on developing strategy reducing the cost of performance estimation. In this work, during the GA process, we adopt the following straightforward and efficient method to estimate the performance of an ANN: first, train a neural network one hundred times on the training dataset; second, do the validation test; measure the neural network's performance according to its fitness of objective function on the test dataset.
TABLE III. COMPARISON OF THE CARBON/EPOXY, GRAPHITE/EPOXY, AND GLASS/EPOXY PROPERTIES

\begin{tabular}{lccccc}
\hline Property & Symbol & Unit & Carbon/Epoxy & Graphite/Epoxy & Glass/Epoxy \\
\hline Longitudinal elastic modulus & $E_{1}$ & $\mathrm{GPa}$ & 116.6 & 181 & 38.6 \\
Traverse elastic modulus & $E_{2}$ & $\mathrm{GPa}$ & 7.67 & 10.3 & 8.27 \\
Major Poisson's ratio & $\sigma_{12}$ & $\mathrm{GPa}$ & 0.27 & 0.28 & 0.26 \\
Shear modulus & $G_{12}$ & $\mathrm{GPa}$ & 7.17 & 4.14 \\
Ultimate longitudinal tensile strength & $\left(\sigma_{1}^{T}\right)$ ult & $\mathrm{MP}$ & 2062 & 1500 & 1062 \\
Ultimate longitudinal compressive strength & $\left(\sigma_{1}^{C}\right)_{u l t}$ & $\mathrm{MP}$ & 1701 & 1500 & 610 \\
Ultimate transverse tensile strength & $\left(\sigma_{2}^{T}\right)$ ult & $\mathrm{MPa}$ & 70 & 40 & 31 \\
Ultimate transverse compressive strength & $\left(\sigma_{2}^{C}\right)$ ult & $\mathrm{MPa}$ & 240 & 246 & 118 \\
Ultimate in-plane shear strength & $\left(\tau_{12}\right)$ ult & $\mathrm{MPa}$ & 105 & 68 & 72 \\
Density & $\rho$ & $\mathrm{g} / \mathrm{cm}^{3}$ & 1.605 & 1.590 & 1.903 \\
Cost & & & 8 & 2.5 & 1 \\
\hline
\end{tabular}

TABLE IV. EXAMPLES OF THE TRAINING DATA

\begin{tabular}{cccc|cc}
\hline & \multicolumn{3}{c}{ Input } & \multicolumn{2}{c}{ Output } \\
\hline \multirow{2}{*}{ Load } & $\begin{array}{c}\text { Laminate } \\
\text { Structure }\end{array}$ & $\begin{array}{c}\text { Material } \\
\text { Property }\end{array}$ & $\begin{array}{c}\text { Failure } \\
\text { Property }\end{array}$ & MS & \multirow{2}{*}{ Tsai-Wu } \\
\hline$-70,-10,-40$, & $90,-90,4,1.27$, & $38.6,8.27,0.26,4.14$, & $1062.0,610.0,31,118,72$, & 0.0102, & 0.0086 \\
$-10,10,0$, & $-86,86,80,1.27$, & $181.0,10.3,0.28,7.17$, & $1500.0,1500,0,40,246,68$, & 0.4026, & 2.5120 \\
$-70,-50,80$, & $-38,38,4,1.27$, & $116.6,7.67,0.27,4.173$, & $2062.0,1701.0,70,240,105$, & 0.0080, & 0.0325 \\
$-70,80,-40$, & $90,-90,48,1.27$, & $38.6,8.27,0.26,4.14$, & $1062.0,610.0,31,118,72$, & 0.0218, & 0.1028 \\
$-20,-30,0$, & $-86,86,60,1.27$, & $181.0,10.3,0.28,7.17$, & $1500.0,1500.0,40,246,68$, & 0.6481, & 0.9512 \\
$0,-40,0$, & $74,-74,168,1.27$, & $181.0,10.3,0.28,7.17$, & $1500.0,1500.0,40,246,68$, & 1.3110, & 3.9619 \\
\hline
\end{tabular}

\section{EXPERIMENT}

In the previous section, we present the details of our strategies for designing an ANN. In this section, we explain the details of the preparation of the training dataset and validation dataset.

\section{A. Dataset Preparation}

For composite material, it is impossible to obtain massive training data from the practical scenario. Therefore, we use classical lamination theory and failure theory, which follows a two-step procedure: first, evaluate the stress and strain according to classic lamination theory; second, substitute them into the corresponding equation to get the strength ratio. Repeat this procedure to yield 14000 points uniformly distributed over the domain space, and define the domain of the corresponding inputs as follows: the range of in-plane loading varies from 0 to 120 ; the range of fiber orientation $\theta$ is from -90 to 90 ; ply thickness $t$ is $1.27 \mathrm{~mm}$, the number of plies ranges $N$ is from 4 to 120. Three different composite material is used in this experiment, as shown in Table III. Table IV shows part of the training data, which are randomly selected from the generated training dataset. To speeds up the learning and accelerate convergence, the input attributes of the dataset are rescaled to between 0 and 1.0 by a linear function.

\section{B. ANN Training and Validation}

The ANN training procedure is carried out by optimizing the multinomial logistic regression objective using mini-batch gradient descent [27] with momentum. The batch size is set to 1000 , momentum to 0.9 . the learning rate is set to $10^{-2}$. The ratio of the training dataset and validation dataset is $70 / 30$, with $70 \%$ of the entire data for training and $30 \%$ for validation.

\section{Genetic Algorithm}

The genetic algorithm involves the evolution of an artificial neural network's topology, activation function, etc. in the optimizing process. The corresponding parameters are as the following. The population is 10 , the percentage of parents in 


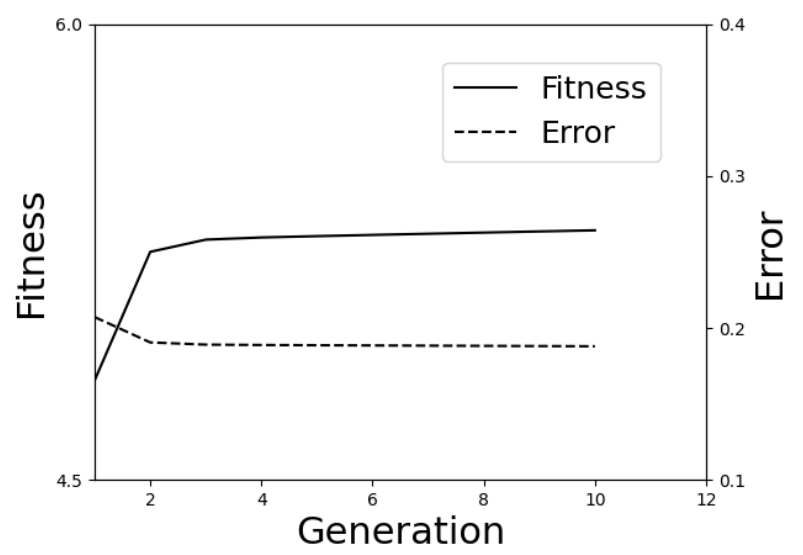

Fig. 8. Fitness and Averaged Sum-of-squares Errors of the Pre-trained Artificial Neural Network as Generations Proceed.

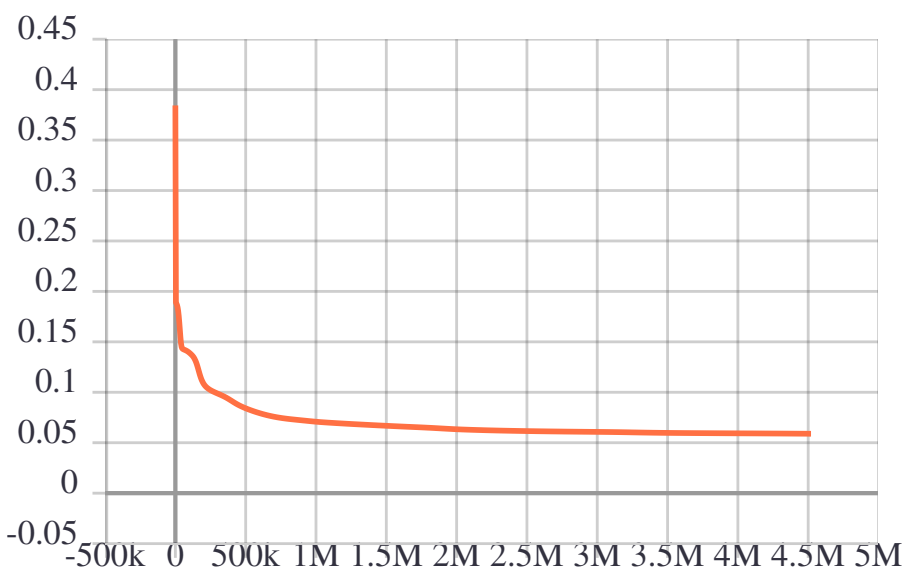

Fig. 9. The illustration of the Behaviour of Fitness on the Training Dataset During the Training Session.

the population is $40 \%$; the strategy of selecting parents is rankbased; the mutation rate of the offspring is 0.3 .

\section{RESUlT AND DiscusSION}

In this work, we propose to use an artificial neural network as an alternative way to compute the strength ratio of composite material instead of a two-step procedure, based on classical lamination and failure theory. Fig. 8 shows the changes of the fitness and error during the evolution procedure. The fitness is obtained through the performance estimation technique of an artificial neural network. As shown in this figure, fitness grows during the initial stage; then, it slowly converges as generation proceeds. It implies genetic algorithm can find a better artificial neural network with the evolution of the number of neurons in the hidden layer, connection relationship, activation functions, and connection weights.

Fig. 9 shows the rest training of the artificial neural network obtained from the GA, which is a pre-trained ANN. Continue to train it with a standard gradient-based descent algorithm until the error converges. The target neural network converges rapidly at first, and further training doesn't reduce the error
TABLE V. ANN PREDICTIONS OF THE TSAI-WU AND MS STRENGTH RATIO With THE NUMBERICAL RESULTS ObTAINED By CLT.

\begin{tabular}{cccc|cc|cc}
\hline & & \multicolumn{2}{c}{ Input } & \multicolumn{4}{c}{ Output } \\
\hline \multirow{2}{*}{ Load } & Laminate & Material & Failure & \multicolumn{2}{c}{ CLT } & \multicolumn{3}{c}{ ANN } \\
& Structure & Property & Property & MS Tsai-Wu & MS Tsai-Wu \\
\hline$-10,40,20$ & $26,-26,168,1.27$ & $116.6,7.67,0.27,4.17$ & $2062.0,1701.0,70,240,105$ & 0.342 & 0.476 & 0.351 & 0.492 \\
$20,-70,-30$ & $10,-10,196,1.27$ & $181.0,10.3,0.28,7.17$ & $1500.0,1500.0,40,246,68$ & 0.653 & 0.489 & 0.612 & 0.445 \\
$60,-20,0$ & $82-82,128,1.27$ & $181.0,10.3,0.28,7.17$ & $1500.0,1500.0,40,246,68$ & 1.663 & 0.112 & 1.673 & 0.189 \\
\hline
\end{tabular}

efficiently. Then, this artificial neural network is used to predict the strength ratio of laminated composite material.

To present the evaluation result of the ANN straightforwardly, several experiment results from the validation dataset are displayed in Table V, which are randomly selected. Comparing the strength ratio outputs based on CLT and ANN from Table $\mathrm{V}$, it is shown that the calculation of strength ratio can be achieved using a two-layer neural network, without the intensive computation of matrix multiplication.

\section{CONCLUSION}

In this paper, an evolutionary artificial neural network model was developed to predict the strength ratio of laminated composite material under in-plane loading. We review the use of genetic algorithms and artificial neural networks as an alternative approach for calculating the strength ratio of an angle ply laminate under in-plane loading. Traditionally, it is obtained through CLT and corresponding failure criteria, such as Maximum Stress theory and Tsai-Wu failure theory.

The main contribution of this work is as follows: 1) propose a two-layer diagram model for designing a sophisticated neural network in simulating the calculation of strength ratio, and use a genetic algorithm to explore the search space; 2) suggest an efficient method to compute the strength ratio instead of adopting the two-step procedure based on classical lamination theory and related failure criteria. Compared with experimentally obtained data, it is demonstrated that ANN is an efficient and simple tool to compute the strength ratio, instead of the complex analytical mathematical model. Our findings underline the practical applicability of ANN on the analysis of composite material.

There are more improvements we can make over the search strategy and application in the area of laminated composite material. The future work is to develop a more sophisticated ANN, which not only can predict the properties for angle ply laminate, but also the other type of laminated composite material.

\section{ACKNOWLEDGMENT}

The work has partly been supported by China Scholarship Council(CSC) under grant no. 201806630112

\section{REFERENCES}

[1] A. Todoroki and R. T. Haftka, "Stacking sequence optimization by a genetic algorithm with a new recessive gene like repair strategy," Composites Part B: Engineering, vol. 29, no. 3, pp. 277-285, 1998.

[2] B. Liu, R. T. Haftka, M. A. Akgün, and A. Todoroki, "Permutation genetic algorithm for stacking sequence design of composite laminates," Computer methods in applied mechanics and engineering, vol. 186, no. 2-4, pp. 357-372, 2000. 
[3] K. Sivakumar, N. Iyengar, and K. Deb, "Optimum design of laminated composite plates with cutouts using a genetic algorithm," Composite Structures, vol. 42, no. 3, pp. 265-279, 1998.

[4] M. Walker and R. E. Smith, "A technique for the multiobjective optimisation of laminated composite structures using genetic algorithms and finite element analysis," Composite structures, vol. 62, no. 1, pp. 123-128, 2003.

[5] C.-C. Lin and Y.-J. Lee, "Stacking sequence optimization of laminated composite structures using genetic algorithm with local improvement," Composite structures, vol. 63, no. 3-4, pp. 339-345, 2004.

[6] J.-H. Kang and C.-G. Kim, "Minimum-weight design of compressively loaded composite plates and stiffened panels for postbuckling strength by genetic algorithm," Composite structures, vol. 69, no. 2, pp. 239246, 2005.

[7] M. Murugan, S. Suresh, R. Ganguli, and V. Mani, "Target vector optimization of composite box beam using real-coded genetic algorithm: a decomposition approach," Structural and Multidisciplinary Optimization, vol. 33, no. 2, pp. 131-146, 2007.

[8] M. Akbulut and F. O. Sonmez, "Optimum design of composite laminates for minimum thickness," Computers \& Structures, vol. 86, no. 21-22, pp. 1974-1982, 2008.

[9] S. Yan, X. Zou, M. Ilkhani, and A. Jones, "An efficient multiscale surrogate modelling framework for composite materials considering progressive damage based on artificial neural networks," Composites Part B: Engineering, vol. 194, p. 108014, 2020. [Online]. Available: https://www.sciencedirect.com/science/article/pii/S1359836820303279

[10] N. Mentges, B. Dashtbozorg, and S. Mirkhalaf, "A micromechanics-based artificial neural networks model for elastic properties of short fiber composites," Composites Part B: Engineering, vol. 213, p. 108736, 2021. [Online]. Available: https://www.sciencedirect.com/science/article/pii/S1359836821001281

[11] F. Lobo, C. F. Lima, and Z. Michalewicz, Parameter setting in evolutionary algorithms. Springer Science \& Business Media, 2007, vol. 54.

[12] Y. Liu and X. Yao, "Evolutionary design of artificial neural networks with different nodes," in Proceedings of IEEE international conference on evolutionary computation. IEEE, 1996, pp. 670-675.

[13] S. Rodzin, O. Rodzina, and L. Rodzina, "Neuroevolution: problems, algorithms, and experiments," in 2016 IEEE 10th International Conference on Application of Information and Communication Technologies (AICT). IEEE, 2016, pp. 1-4.

[14] T. N. Massard, "Computer sizing of composite laminates for strength," Journal of reinforced plastics and composites, vol. 3, no. 4, pp. 300345, 1984.
[15] J. Reddy and A. Pandey, "A first-ply failure analysis of composite laminates," Computers \& Structures, vol. 25, no. 3, pp. 371-393, 1987.

[16] C. Fang and G. S. Springer, "Design of composite laminates by a monte carlo method," Journal of composite materials, vol. 27, no. 7, pp. 721753, 1993.

[17] A. Soeiro, C. C. António, and A. T. Marques, "Multilevel optimization of laminated composite structures," Structural optimization, vol. 7, no. 1-2, pp. 55-60, 1994.

[18] J. L. Pelletier and S. S. Vel, "Multi-objective optimization of fiber reinforced composite laminates for strength, stiffness and minimal mass," Computers \& structures, vol. 84, no. 29-30, pp. 2065-2080, 2006.

[19] P. Jadhav and P. R. Mantena, "Parametric optimization of grid-stiffened composite panels for maximizing their performance under transverse loading," Composite structures, vol. 77, no. 3, pp. 353-363, 2007.

[20] S. Omkar, R. Khandelwal, S. Yathindra, G. N. Naik, and S. Gopalakrishnan, "Artificial immune system for multi-objective design optimization of composite structures," Engineering Applications of Artificial Intelligence, vol. 21, no. 8, pp. 1416-1429, 2008.

[21] A. Choudhury, S. Mondal, and S. Sarkar, "Failure analysis of laminated composite plate under hygro-thermo mechanical load and optimisation," International Journal of Applied Mechanics and Engineering, vol. 24, no. 3, pp. 509-526, 2019.

[22] R. Watkins and A. Morris, "A multicriteria objective function optimization scheme for laminated composites for use in multilevel structural optimization schemes," Computer Methods in Applied Mechanics and Engineering, vol. 60, no. 2, pp. 233-251, 1987.

[23] P. Martin, "Optimum design of anisotropic sandwich panels with thin faces," Engineering optimization, vol. 11, no. 1-2, pp. 3-12, 1987.

[24] C. M. Soares, V. F. Correia, H. Mateus, and J. Herskovits, "A discrete model for the optimal design of thin composite plate-shell type structures using a two-level approach," Composite structures, vol. 30, no. 2 , pp. 147-157, 1995.

[25] G. Cybenko, "Approximation by superpositions of a sigmoidal function," Mathematics of control, signals and systems, vol. 2, no. 4, pp. 303-314, 1989.

[26] B. Baker, O. Gupta, R. Raskar, and N. Naik, "Accelerating neural architecture search using performance prediction," arXiv preprint arXiv:1705.10823, 2017.

[27] Y. LeCun, B. Boser, J. S. Denker, D. Henderson, R. E. Howard, W. Hubbard, and L. D. Jackel, "Backpropagation applied to handwritten zip code recognition," Neural computation, vol. 1, no. 4, pp. 541-551, 1989. 